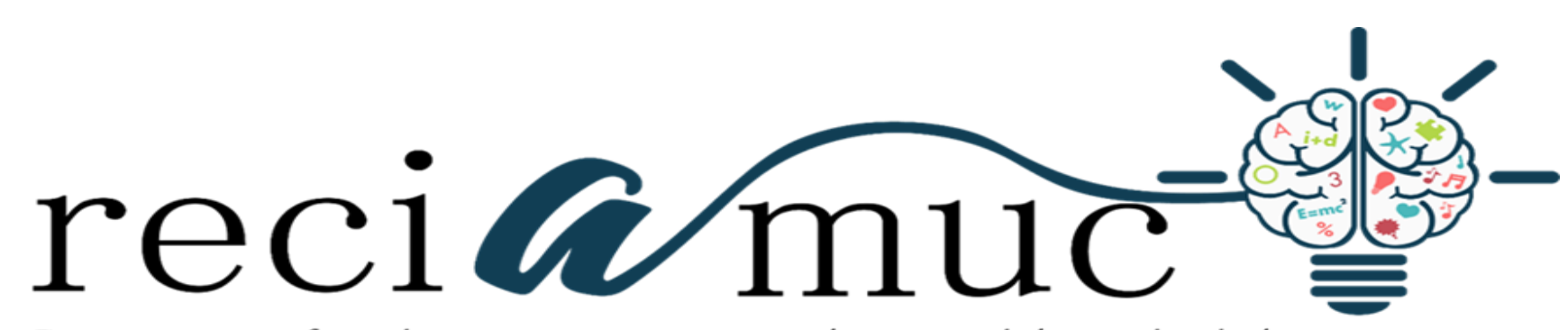

Revista científica de investigación actualización del mundo de las ciencias

Ángela Lilibeth Peñafiel Bernal a; Javier Alejandro Mera Cedeño ${ }^{\text {b; }}$ Andrea Isabel Vallejo Maquilón ${ }^{\text {c; }}$ María Belén Meza Rivas ${ }^{\text {d }}$

Consideraciones para la intervención quirúrgica frente a afecciones que provocan apendicitis aguda

Considerations for surgical intervention against conditions that cause acute appendicitis

Revista Científica de Investigación actualización del mundo de las Ciencias. Vol. 3 núm., 3, julio, ISSN: 2588-0748, 2018, pp.1227-1247

DOI: $10.26820 /$ reciamuc/3.(3).julio.2019.1227-1247

URL: http://reciamuc.com/index.php/RECIAMUC/article/view/379

Código UNESCO: 3205 Medicina Interna

Tipo de Investigación: Artículo de Investigación

(C) RECIAMUC; Editorial Saberes del Conocimiento, 2019

Recibido: 28/04/2019

Aceptado: 19/05/2019

Publicado: 01/07/2019

Correspondencia: angelitabpbm@gmail.com

a. Médico Cirujano; Hospital IESS Manta; angelitabpbm@gmail.com; Manta - Ecuador.

b. Médico Cirujano; Hospital IESS Manta; javieralejandromera@ hotamail.com; Manta - Ecuador.

c. Médica Cirujana; Hospital IESS Portoviejo; andreavallejo_90@ @otmail.com; Portoviejo - Ecuador.

d. Médica Cirujana; Hospital IESS Portoviejo; belenmeza1205@gmail.com; Portoviejo - Ecuador. 


\section{Consideraciones para la intervención quirúrgica frente a afecciones que provocan apendicitis aguda}

Vol. 3, núm. 3., (2019)

Ángela Lilibeth Peñafiel Bernal; Javier Alejandro Mera Cedeño; Andrea Isabel Vallejo

Maquilón; María Belén Meza Rivas

\section{RESUMEN}

El dolor abdominal es uno de los principales motivos de consulta, entre los cuales uno de los diagnósticos diferenciales es la apendicitis aguda, sin embargo, no a todos los pacientes se les diagnóstica oportunamente esta enfermedad, trayendo como consecuencia que éstos se compliquen. Entiéndase con esto que, el diagnóstico de esta patología es netamente clínico ya que depende de una buena anamnesis y un buen examen físico, la misma ha llevado, a un elevado número de ingresos hospitalarios, los cuales, en muchas ocasiones han sido desarrollados, sin llegar a un diagnóstico preciso por demoras en la atención o por confusión clínica, las cuales surgen a raíz de la similitud que este cuadro médico tiene con patologías tales como: neumonía basal derecha, infecciones urinarias, gastrointestinales, intoxicaciones; situación ésta que ha generado un marcado interés porque la ciencia encuentre un método que permita con certeza hacer un diagnóstico de apendicitis aguda más tempranamente. En virtud de esto, han surgido nuevos métodos dirigidos a la identificación temprana de este tipo de afección médica; destacándose entre ellas la escala RIPASA desarrollada, en el sudeste de Asia en el hospital RIPAS con el fin de adecuar a pacientes con otro tipo de alimentación (alta en fibra) y que conlleva un factor protector, la cual ya fue validad y presenta una sensibilidad del $98 \%$ y especificidad del $83 \%$ a diferencia de la escala Solís - Mena realizada por médicos mexicanos con población del norte del país la cual fue realizada con 100 pacientes y de acuerdo al reporte de SOLIS Y COLS se presenta mayor sensibilidad y especificidad (sensibilidad 92\% especificidad 93\%)que la escala de ALVARADO, sin embargo esta escala no ha sido validada; planteamientos que han llevado a desarrollar artículos que busquen establecer las consideraciones éticas para obtener un adecuado diagnóstico de apendicitis aguda.

Palabras Claves: Dolor Abdominal; Apendicitis Aguda; Tratamiento Quirúrgico; Escala RIPASA; Escala SOLIS Y COLS; Escala de ALVARADO. 


\title{
Consideraciones para la intervención quirúrgica frente a afecciones que provocan apendicitis aguda
} Vol. 3, núm. 3., (2019) Ángela Lilibeth Peñafiel Bernal; Javier Alejandro Mera Cedeño; Andrea Isabel Vallejo Maquilón; María Belén Meza Rivas

\begin{abstract}
Abdominal pain is one of the main reasons for consultation, among which one of the differential diagnoses is acute appendicitis, however, not all patients are opportunely diagnosed with this disease, resulting in complications. Understand with this that, the diagnosis of this pathology is purely clinical since it depends on a good history and a good physical examination, it has led to a high number of hospital admissions, which, in many cases have been developed, without arrive at an accurate diagnosis due to delays in care or clinical confusion, which arise as a result of the similarity that this medical condition has with pathologies such as: right basal pneumonia, urinary, gastrointestinal infections, intoxications; This situation has generated a marked interest because science finds a method that allows for a diagnosis of acute appendicitis earlier. By virtue of this, new methods have emerged for the early identification of this type of medical condition; standing out among them the developed RIPASA scale, in Southeast Asia in the RIPAS hospital in order to adapt patients with another type of diet (high in fiber) and that entails a protective factor, which was already valid and has a sensitivity of $98 \%$ and specificity of $83 \%$ unlike the Solís - Mena scale carried out by Mexican doctors with a population from the north of the country which was performed with 100 patients and according to the SOLIS Y COLS report there is a greater sensitivity and specificity (sensitivity $92 \%$ specificity 93\%) than the ALVARADO scale, however this scale has not been validated; approaches that have led to the development of articles that seek to establish ethical considerations to obtain an adequate diagnosis of acute appendicitis.
\end{abstract}

Key Words: Abdominal Pain; Acute appendicitis; Surgical Treatment; RIPASA scale; SOLIS AND COLS scale; ALVARADO scale. 


\section{Consideraciones para la intervención quirúrgica frente a afecciones que provocan apendicitis aguda}

Vol. 3, núm. 3., (2019)

Ángela Lilibeth Peñafiel Bernal; Javier Alejandro Mera Cedeño; Andrea Isabel Vallejo

Maquilón; María Belén Meza Rivas

\section{Introducción.}

La apendicitis aguda es la principal causa de abdomen agudo quirúrgico, su frecuencia predomina en el grupo de edad comprendido entre los 10 y 30 años de edad, su diagnóstico es clínico, realizado con una historia clínica bien desarrollada y una exploración física completa. Clásicamente esta sintomatología tiende a confundir la evolución de esta enfermedad; especialmente, en circunstancias especiales, en las que se puede presentar un cuadro atípico o enmascarado principalmente en embarazadas, niños ancianos y principalmente por tratamiento médico previo.

Esta enfermedad ha sido catalogada como una de las patologías con mayor porcentaje de incidencia y causa de hospitalización en países latinoamericanos, esta alteración biológica se presenta de manera general, a través de un dolor agudo en el abdomen, a nivel de clínica es registrada como la más frecuente emergencia quirúrgica abdominal y la cuarta operación más frecuente en la cirugía intra - abdominal. Por ello, su diagnóstico debe ser manejado, de manera profesional, bajo un estricto conocimiento de anatomía, fisiopatología y la clínica, así como de las etapas que se producen en el desarrollo de esta enfermedad, pues, si bien, sus síntomas evolucionan en forma clásica, a veces hay cuadros atípicos que ponen al paciente en riesgo de padecer complicaciones. (Selis, 2016).

Como consecuencia de esto, se ha podido observar que existe un alto porcentaje de pacientes que se retrasan 72 o más horas desde el inicio de los síntomas hasta el acto operatorio. Estos factores de demora, se ven condicionados por el hecho entre muchas causas, de que los pacientes no recurren a tiempo a los servicios de salud a que les realicen el diagnóstico de su padecimiento, lo que establece que estas personas, tengan un retraso considerable, deben ser intervenidos a tiempo, lo que los lleva a formar parte de un 2,5\% de la población en situación de riesgo clínico a padecer complicaciones médicas. (Cerpa, 2015). 


\section{Consideraciones para la intervención quirúrgica frente a afecciones que provocan apendicitis aguda}

Vol. 3, núm. 3., (2019)

Ángela Lilibeth Peñafiel Bernal; Javier Alejandro Mera Cedeño; Andrea Isabel Vallejo

Maquilón; María Belén Meza Rivas

En términos generales, la apendicitis proviene de un proceso evolutivo, secuencial, de allí las diversas manifestaciones clínicas y anatomo - patológicas que suele encontrar el cirujano y que dependerán fundamentalmente del momento o fase de la enfermedad en que es abordado el paciente, de allí que se consideren los siguientes estadios: apendicitis congestiva o catarral, apendicitis flemonosa o supurativa, apendicitis gangrenosa o necrótica, apendicitis perforada. (Penza, 2016).

Por consiguiente, se logra entender que la apendicitis es la cirugía no electiva más común. Para el año 2017 fueron diagnosticadas 180000 de estas operaciones en Ecuador y 30000 casos por año en el Perú. Es muy frecuente en los hombres. Se da sobre todo en los adultos, entre los 20 y 30 años. El $1 \%$ de los afectados mueren, pero la mortalidad entre niños es más alta: 4 al 8\%. El diagnóstico adecuado de esta alteración depende mucho de una buena historia clínica, un buen examen físico y una buena interpretación de los exámenes auxiliares, siendo su única cura el proceso quirúrgico. (Lenín, 2017).

Un diagnóstico certero de esta enfermedad va a depender de la ubicación del apéndice. Si este se encuentra retro cecalmente, el dolor comenzará en alguna parte del hemi abdomen derecho. Si el apéndice es largo, yendo más allá de la línea media, podría haber dolor en el cuadrante inferior izquierdo. Por lo general habrá anorexia y nausea, así como vómitos. Rara vez hay vómitos antes del dolor. La presencia de apetito hará dudar del diagnóstico. Existe una cronología en los síntomas: dolor, anorexia, incremento de la sensibilidad, fiebre y aumentos de leucocitos en sangre. Una alteración de esta cronología permitirá dudar del diagnóstico. (Lenín Ob cit).

Para el diagnóstico diferencial, son utilizados los exámenes en el laboratorio. Por lo general se pide un hemograma y un examen de orina. La leucocitosis entre los 12000 y $18000 \mathrm{~mm} 3$ con predominio de neutrófilos sirve para confirmar la presencia de esta enfermedad; por lo general, son considerados valores superiores a 18 000, los cuales sugerirían la presencia de apendicitis con perforación; por su parte, el examen urinario sirve para descartar la posibilidad de infección urinaria; no obstante, se debe tener presente que, hasta el 33\% de 


\section{Consideraciones para la intervención quirúrgica frente a afecciones que provocan apendicitis aguda}

Vol. 3, núm. 3., (2019)

Ángela Lilibeth Peñafiel Bernal; Javier Alejandro Mera Cedeño; Andrea Isabel Vallejo

Maquilón; María Belén Meza Rivas

los pacientes presentarán piuria y hematuria sin bacteriuria, debido a que tanto el uréter y la vejiga están cerca del apéndice. (Castro, 2016).

La apendicitis aguda es una urgencia quirúrgica frecuente con una incidencia anual de 1. por 1000 personas por año. la población blanca es la más afectada que en otros grupos. Por causas desconocidas, la apendicitis ocurre con más frecuencia durante el verano. En relación con la edad se señala que entre O y 12 se presenta en un 18\%, entre 13 y 39 años en un $70 \%$ después de los 40 años en un 12\%. (Castro Ob cit)

Esta enfermedad no respeta ni raza ni condición social, puede ocurrir desde el nacimiento hasta la ancianidad, no hay tiempo o condiciones en las cuales el padecimiento no pueda presentarse. El riesgo de apendicitis durante toda la vida es cerca del 7\%. La morbilidad y mortalidad relacionadas con la apendicitis siguen siendo altas, a pesar del énfasis en la intervención quirúrgica rápida.

Por todo esto, es de gran importancia que el médico tratante tenga la oportunidad de establecer de manera efectiva la secuencia de aparición de los síntomas tiene gran importancia en el diagnóstico diferencial, en la mayoría de los pacientes el primer síntoma es la anorexia, seguido de dolor en abdomen que es continuo, de intensidad moderada, aumenta con la tos, movimientos respiratorios o deambulación, que a su vez va seguido de vómitos, a toda ésta secuencia de sintomatología se le llama cronología apendicular de Murphy; aunque la anorexia se encuentra en la mayoría de pacientes operados de apendicitis, en algunas oportunidades el paciente puede tener hambre.

Por otra parte, existen otros puntos dolorosos con los cuales se pretende correlacionar las manifestaciones clínicas, como los son el Signo de Rovsing, la Hiperestesia cutánea de Sherren, Punto de Lanz, Punto de Lecene, Punto de Morris, Signo del obturador: Se produce por rotación interna del miembro inferior derecho que provoca dolor en la apendicitis pelviana, el examen de Tacto rectal, el cual debe de hacerse de rutina, aunque en muchas ocasiones este puede ser negativo, sobre todo en algunos casos donde se pueden encontrar un fondo de saco de Douglas sumamente doloroso, sobre todo cuando hay perforación del 


\section{Consideraciones para la intervención quirúrgica frente a afecciones que provocan apendicitis aguda}

Vol. 3, núm. 3., (2019)

Ángela Lilibeth Peñafiel Bernal; Javier Alejandro Mera Cedeño; Andrea Isabel Vallejo

Maquilón; María Belén Meza Rivas

apéndice y derrame purulento. Entre otras técnicas que serán analizadas a lo largo del presente artículo.

\section{Tipo de Investigación.}

Dentro de toda práctica investigativa, se precisan acciones de carácter metodológico mediante las cuales, se logra conocer y proyectar los eventos posibles que la determinan, así como las características que hacen del acto científico un proceso interactivo ajustado a una realidad posible de ser interpretada. En este sentido, se puede decir, que la presente investigación corresponde al tipo documental, definido por (Dávila, 2012), "se ocupa del estudio de problemas planteados a nivel teórico, la información requerida para abordarlos se encuentra básicamente en materiales impresos, audiovisuales y /o electrónicos". (p.41).

En consideración a esta definición, la orientación metodológica permitió la oportunidad de cumplir con una serie de actividades inherentes a la revisión y lectura de diversos documentos donde se encontraron ideas explicitas relacionadas con los tópicos encargados de identificar a cada característica insertada en el estudio. Por lo tanto, se realizaron continuas interpretaciones con el claro propósito de revisar aquellas apreciaciones o investigaciones propuestas por diferentes investigadores, para luego dar la respectiva argumentación a los planteamientos, en función a las necesidades encontradas en la indagación.

\section{Fuentes Documentales.}

El análisis correspondiente a las características que predomina en el tema seleccionado, llevan a incluir diferentes fuentes documentales encargadas de darle el respectivo apoyo y en ese sentido cumplir con la valoración de los hechos a fin de generar nuevos criterios que sirven de referencia a otros procesos investigativos. Para (Arias, 2010), las fuentes documentales incorporadas en la investigación documental o bibliográfica, "representa la suma de materiales sistemáticos que son revisados en forma rigurosa y profunda para llegar a un análisis del fenómeno". (p.41). Por lo tanto, se procedió a cumplir con la realización de una lectura previa determinada por encontrar aquellos aspectos estrechamente vinculados con las "Consideraciones para la intervención 


\section{Consideraciones para la intervención quirúrgica frente a afecciones que provocan apendicitis aguda}

Vol. 3, núm. 3., (2019)

Ángela Lilibeth Peñafiel Bernal; Javier Alejandro Mera Cedeño; Andrea Isabel Vallejo

Maquilón; María Belén Meza Rivas

quirúrgica frente a afecciones que provocan la Apendicitis Aguda" para luego explicar mediante un desarrollo las respectivas apreciaciones generales de importancia.

\section{Técnicas para la Recolección de la Información.}

La conducción de la investigación para ser realizada en función a las particularidades que determinan a los estudios documentales, tiene como fin el desarrollo de un conjunto de acciones encargadas de llevar a la selección de técnicas estrechamente vinculadas con las características del estudio. En tal sentido, (Arias Ob cit) refiere, que es "una técnica particular para aportar ayuda a los procedimientos de selección de las ideas primarias y secundarias”. (p. 71).

Por ello, se procedió a la utilización del subrayado, resúmenes, fichaje, como parte básica para la revisión y selección de los documentos que presentan el contenido teórico. Es decir, que mediante su aplicación de estas técnicas se pudo llegar a recoger informaciones en cuanto a la revisión bibliográfica de los diversos elementos encargados de orientar el proceso de investigación. Tal como lo expresa, (Arias Ob cit) "las técnicas documentales proporcionan las herramientas esenciales y determinantes para responder a los objetivos formulados y llegar a resultados efectivos" (p. 58). Es decir, para responder con eficiencia a las necesidades investigativas, se introdujeron como técnica de recolección el método inductivo, que hizo posible llevar a cabo una valoración de los hechos de forma particular para llegar a la explicación desde una visión general.

Asimismo, se emplearon las técnicas de análisis de información para la realización de la investigación que fue ejecutada bajo la dinámica de aplicar diversos elementos encargados de determinar el camino a recorrer por el estudio, según, (Arias, Ob cit) las técnicas de procesamiento de datos en los estudios documentales "son las encargadas de ofrecer al investigador la visión o pasos que debe cumplir durante su ejercicio, cada una de ellas debe estar en correspondencia con el nivel a emplear" (p. 123). Esto indica, que, para llevar a cabo el procesamiento de los datos obtenidos, es necesario establecer las técnicas que serán seleccionadas, destacándose en este caso, de manera particular: fichas de resumen, textual, registros descriptivos entre otros, los mismos se deben ajustar al nivel que ha sido seleccionado. 


\section{Consideraciones para la intervención quirúrgica frente a afecciones que provocan apendicitis aguda}

Vol. 3, núm. 3., (2019)

Ángela Lilibeth Peñafiel Bernal; Javier Alejandro Mera Cedeño; Andrea Isabel Vallejo

Maquilón; María Belén Meza Rivas

\section{Resultados.}

\section{Apéndice Cecal.}

La apéndice representa un órgano, que de manera normal está situado en la fosa iliaca derecha y su fondo corresponde al ángulo diedro que esta fosa forma con la pared anterior del abdomen, siendo su dirección, oblicua, hacia abajo, adentro y adelante. Sin embargo, algunas veces se haya colocado más arriba, dispuesto transversalmente en la región lumbar (situación alta del ciego). En otras ocasiones se encuentra por dentro del Psoas e invade la cavidad de la pelvis menor (situación baja del ciego).

Se encuentra fijo por el peritoneo, que lo rodea en toda su extensión, por su continuidad con el colon ascendente, así como por la conexión que tiene el intestino delgado. El meso colon ascendente lo fija a la pared posterior del abdomen, pues, aunque el ciego no tenga meso, la porción inicial del meso colon ascendente, que se inserta en la fosa iliaca y está formado por las dos hojas de la extremidad inferior del mesenterio, tiene tal relación con el ciego, que origina un meso, a veces llamado ligamento del ciego o meso ciego. 


\section{Consideraciones para la intervención quirúrgica frente a afecciones que provocan apendicitis aguda}

Vol. 3, núm. 3., (2019)

Ángela Lilibeth Peñafiel Bernal; Javier Alejandro Mera Cedeño; Andrea Isabel Vallejo

Maquilón; María Belén Meza Rivas

\section{Figura $\mathbf{N}^{\circ}$ 1. Apendicitis Aguda}

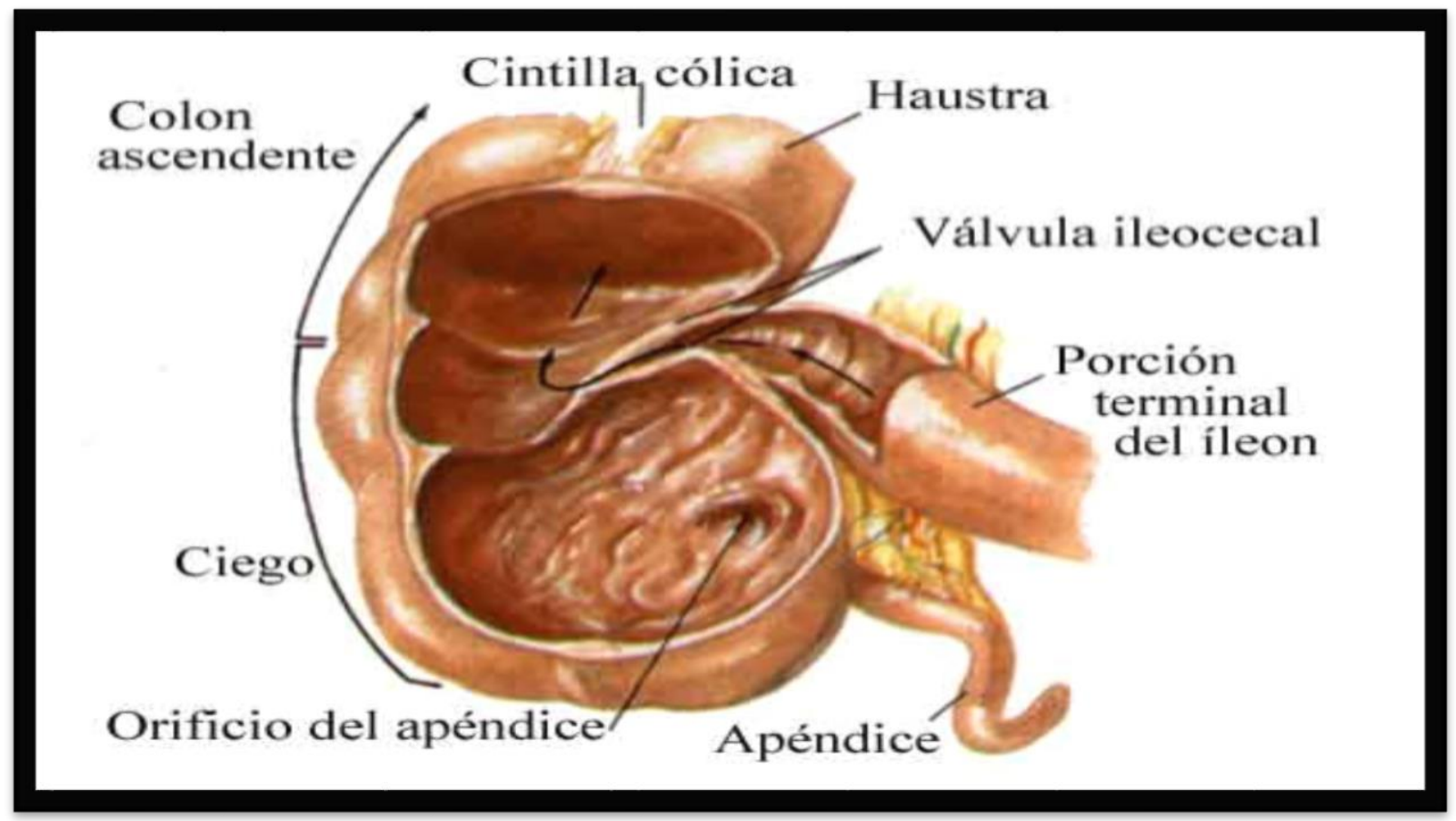

Fuente: (Lenín 2016).

El apéndice en el hombre es una estructura que no tiene función útil, se le considera como la amígdala linfática del abdomen por sus numerosos folículos. El apéndice vermicular (APPENDIX o PROCESIS VERMIFORMTS). Mide 6 a $8 \mathrm{cms}$., de longitud (a veces hasta $20 \mathrm{cms}$ ) suele tener un diámetro de 0.5 a $1 \mathrm{~cm}$. Por lo regular es un poco flexuoso y es muy movible. Este órgano, secreta de 1 a $2 \mathrm{ml}$, de un líquido que contiene, calcio, fosfatos y probablemente algunas enzimas.

Tiene contracciones intermitentes, irregulares, con las que expulsa su contenido al ciego. Posee un mesenterio corto llamado meso apéndice por el recibe vasos sanguíneos, nervios y vasos linfáticos. Suele estar situado directamente en el extremo de la cintilla muscular anterior del colon. A veces se encuentra detrás del ciego en posición llamada reirocecal. En ocasiones queda envuelto en bridas fibrosas congénitas que pueden producir torsión, ensortijamiento o angulación brusca. Estas posiciones defectuosas, en determinadas circunstancias clínicas pueden causar dolor, pues la luz apendicular angulada puede ser distendida por heces gas y originar dolor de tipo eólico. 


\section{Consideraciones para la intervención quirúrgica frente a afecciones que provocan apendicitis aguda}

Vol. 3, núm. 3., (2019)

Ángela Lilibeth Peñafiel Bernal; Javier Alejandro Mera Cedeño; Andrea Isabel Vallejo

Maquilón; María Belén Meza Rivas

La apendicitis, representa un proceso inflamatorio bacteriano del apéndice cecal. El factor causal predominante en el desarrollo de la patología es la obstrucción de la luz, la causa usual de ésta es la obstrucción por fecalitos, otros menos frecuentes son la hipertrofia del tejido linfoide e impacto de semillas y gusanos intestinales. La prolongación de las horas de evolución en más de 25 horas tiene una relación con la presencia de heridas contaminadas y sucias, lo cual es evidente en los resultados quirúrgicos, y es un factor de riesgo que favorece la aparición de infección de sitio operatorio. La ocurrencia de una infección o falla post operatoria en la herida requiere de la coexistencia de algunos factores; inoculación suficiente del germen y disminución en los mecanismos de defensa y alteraciones en las células. Entre sus síntomas más comunes se encuentran:

Abdomen agudo. Es un cuadro abdominal de presentación brusca caracterizado por un conjunto de signos y síntomas generalmente alarmantes, provocada por enfermedades de etiología diversas.

Apendicitis. Es la inflamación del apéndice vermicular del ciego, es una enfermedad aguda, frecuente y de importancia práctica extraordinaria.

Síntomas y Signos Presentes en la Apendicitis (Sensibilidad Y Especificidad

El diagnóstico de la apendicitis aguda continúa siendo clínico, una historia clínica bien desarrollada, así como una buena exploración física dan el diagnóstico en la mayoría de los casos. Cuando el diagnóstico se retrasa, el aumento de la morbimortalidad aumenta considerablemente; bajo esta situación una presentación atípica de apendicitis puede presentarse en menores de dos o mayores de 60 años incluyen en este mismo rubro de cuadros atípicos las presentaciones anatómicas alteradas o modificadas anatómicamente como son: el embarazo, un apéndice largo que se localiza en la fosa ilíaca derecha o un apéndice con localización sub-hepática o simplemente el situs inversus.

En la etapa inicial de la apendicitis el dolor aumenta en el transcurso de 12 a 24 horas y se presenta en $95 \%$ de los pacientes con esta entidad. El dolor es mediado por fibras viscerales aferentes del dolor; se caracteriza por ser poco localizado en epigastrio o a nivel periumbilical, ocasionalmente 


\section{Consideraciones para la intervención quirúrgica frente a afecciones que provocan apendicitis aguda}

Vol. 3, núm. 3., (2019)

Ángela Lilibeth Peñafiel Bernal; Javier Alejandro Mera Cedeño; Andrea Isabel Vallejo

Maquilón; María Belén Meza Rivas

como si fuera un calambre que no mejora con las evacuaciones, el reposo o al cambio de posición. En esta etapa más de $90 \%$ de los pacientes adultos presentan anorexia al igual que $50 \%$ de los pacientes pediátricos.

Un 60\% presentarán náusea. Salvo esta excepción en los adultos: un paciente que presenta apetito, debe hacer dudar del diagnóstico. Las evacuaciones diarreicas en los adultos son raras, siendo más común en los niños. Seis a 12 horas de iniciado el cuadro la inflamación del apéndice se extiende a los órganos que lo rodean y al peritoneo parietal motivo por el cual el dolor es localizado en el punto de Mc Burney. Un 25\% de los pacientes presentan dolor localizado en el cuadrante inferior derecho desde el inicio del cuadro clínico sin presentar síntomas viscerales. J.B. Murphy fue el primero en enfatizar el orden de ocurrencia de la sintomatología: Dolor, anorexia, aumento de la sensibilidad, fiebre y leucocitosis. De igual manera (Lenín 2016).

Comentó:

La sintomatología se presenta en la mayoría de los casos y cuando el orden varía, el diagnóstico debe ser cuestionado. Como en todo hay excepciones; sin embargo, si la náusea o la fiebre se presentan primero, el diagnóstico no es apendicitis. (p.16).

Por todo esto, el diagnóstico de la apendicitis aguda debe basarse en una la inspección general, en la misma, el paciente se mantiene en posición antiálgica, la elevación de la temperatura de 37.5 a $38{ }^{\circ} \mathrm{C}$ es común; sin embargo, de 20-50\% de los pacientes mantienen una temperatura normal. La palpación abdominal muestra rebote positivo, resistencia abdominal voluntaria e involuntaria, en algunas condiciones se puede palpar un plastrón. En las mujeres el tacto rectal y vaginal son mandatorios, ya que son necesarios para el diagnóstico diferencial. Dentro de las maniobras, puntos dolorosos y signos descritos se encuentran los siguientes:

Punto doloroso de Mc Burney: Punto doloroso a la palpación en la unión del tercio medio con el inferior al trazar una línea imaginaria entre el ombligo y la cresta ilíaca derecha. 


\section{Consideraciones para la intervención quirúrgica frente a afecciones que provocan apendicitis aguda}

Vol. 3, núm. 3., (2019) Ángela Lilibeth Peñafiel Bernal; Javier Alejandro Mera Cedeño; Andrea Isabel Vallejo Maquilón; María Belén Meza Rivas

Punto de Lanz: Punto doloroso en la convergencia de la línea interespinal con el borde externo del músculo recto anterior derecho. Se asocia con la ubicación en hueco pélvico del apéndice.

Signo de Blumberg: Dolor en fosa ilíaca derecha a la descompresión. Se presenta en $80 \%$ de los casos.

Signo de Rovsing: Dolor en fosa ilíaca derecha al comprimir la fosa ilíaca izquierda, es explicado por el desplazamiento de los gases por la mano del explorador del colon descendente hacia el transverso, colon ascendente y ciego, que al dilatarse se moviliza produciendo dolor en el apéndice inflamado

Signo del obturador: Se flexiona la cadera y se coloca la rodilla en ángulo recto, realizando una rotación interna de la extremidad inferior, lo que causa dolor en caso de un apéndice de localización pélvica.

Signo de Talo percusión: Dolor en fosa ilíaca derecha con paciente en decúbito dorsal al elevar ligeramente el miembro pélvico derecho y golpear ligeramente en el talón (5)

Signo de Sumner: Defensa involuntaria de los músculos de la pared abdominal sobre una zona de inflamación intraperitoneal. Es más objetivo que el dolor a la presión y se presenta en 90\% de los casos.

Signo de Blumberg: Dolor en fosa ilíaca derecha a la descompresión. Se presenta en $80 \%$ de los casos.

Signo de Mussy: Dolor a la descompresión en cualquier parte del abdomen. Es un signo tardío de apendicitis ya que se considera en este momento una apendicitis fase IV.

Signo del Psoas: Se apoya suavemente la mano en la fosa ilíaca derecha hasta provocar un suave dolor y se aleja hasta que el dolor desaparezca, sin retirarla, se le pide al enfermo que sin doblar la rodilla levante el miembro inferior derecho; el músculo psoas aproxima sus inserciones y ensancha 


\section{Consideraciones para la intervención quirúrgica frente a afecciones que provocan apendicitis aguda}

Vol. 3, núm. 3., (2019)

Ángela Lilibeth Peñafiel Bernal; Javier Alejandro Mera Cedeño; Andrea Isabel Vallejo

Maquilón; María Belén Meza Rivas

su parte muscular movilizando el ciego y proyectándolo contra la mano que se encuentra apoyada en el abdomen, lo que provoca dolor. Es sugestivo de un apéndice retrocecal.

Tabla $\mathbf{N}^{\circ}$ 1. Síntomas y signos presentes en la apendicitis (sensibilidad y especificidad)

\begin{tabular}{|c|c|c|}
\hline Hallazgos & Sensibilidad \% & Especificidad \% \\
\hline \multicolumn{3}{|l|}{ Signos } \\
\hline Fiebre & 67 & 69 \\
\hline Defensa muscular & $39-74$ & $57-84$ \\
\hline Rebote & 63 & 69 \\
\hline Rovsing & 68 & 58 \\
\hline Psoas & 16 & 95 \\
\hline \multicolumn{3}{|l|}{ Sintomas } \\
\hline $\begin{array}{l}\text { Dolor en fosa iliaca } \\
\text { derecha }\end{array}$ & 81 & 53 \\
\hline Nausea & $58-68$ & $37-41$ \\
\hline Vomito & $49-51$ & $45-69$ \\
\hline $\begin{array}{l}\text { Inicio de dolor antes del } \\
\text { vomito. }\end{array}$ & 100 & 64 \\
\hline Anorexia & 84 & 66 \\
\hline
\end{tabular}

Fuente: (Lenín 2016).

Por consiguiente, cuando se sospecha apendicitis, antes de iniciar la palpación del abdomen se pide al paciente que tosa e indique con un dedo el punto más doloroso. Si el punto más doloroso está en la fosa ilíaca derecha, entonces se debe buscar el signo de Rovsing que consiste en la aparición de dolor en FID al comprimir la fosa ilíaca izquierda. Este signo indica por sí solo la presencia de un proceso inflamatorio en la fosa ilíaca derecha, aunque no es específico de apendicitis. 


\section{Consideraciones para la intervención quirúrgica frente a afecciones que provocan apendicitis aguda}

Vol. 3, núm. 3., (2019)

Ángela Lilibeth Peñafiel Bernal; Javier Alejandro Mera Cedeño; Andrea Isabel Vallejo

Maquilón; María Belén Meza Rivas

La palpación del abdomen siempre se practica comenzando sobre los cuadrantes menos dolorosos y avanzando a los más dolorosos y de la superficie a la profundidad. En los cuadros iniciales se encuentra dolor a la palpación profunda en fosa ilíaca derecha. Más tarde puede aparecer contractura de la pared abdominal, es decir, defensa involuntaria, la cual es más intensa cuanto más cerca se encuentre al proceso inflamatorio de la pared abdominal anterior.

En presencia de peritonitis localizada o generalizada, es posible encontrar dolor de rebote, es decir, a la descompresión brusca. Este es el signo de Blumberg, que es de significación definitiva cuando se encuentra presente. Sin embargo, debe ser muy bien evaluado dada la facilidad con que se interpreta en forma equivocada. La palpación puede descubrir también una masa, la cual es signo de un plastrón o de un absceso, es decir, de un proceso localizado. Finalmente, cuando el apéndice se perfora, el paciente empeora de inmediato.

Se torna más febril, aumentan el pulso y la temperatura y el estado general desmejora. Si el proceso no se localiza por epiplón o asas, aparece distensión abdominal por compromiso difuso del peritoneo: es la peritonitis generalizada franca. En el 80\% de los casos el apéndice se perfora antes de las 36 horas de iniciados los síntomas. Cuando ya existe peritonitis, el paciente se mantiene lo más quieto posible para no despertar dolor. La tos y los movimientos agravan la sensación dolorosa, por lo cual el paciente se moviliza con una precaución característica. Al toser se sostiene la fosa ilíaca mientras se queja de dolor.

Signos Clínicos: Los signos clínicos encontrados en un paciente con apendicitis pueden ser desde levemente sospechosos hasta un estado muy florido de signos abdominales.

- Facies: Al inicio no presenta nada de particular, aunque algunas veces la expresión facial refleja disconfort y aprehensión. Cuando el cuadro es compatible con perforación apendicular y peritonitis, la facies puede ser tóxica pálida y con tinte terroso (facies peritonítica).

- Posición: En las primeras horas el paciente prefiere el reposo en posición dorsal; cuando los síntomas se localizan en fosa ilíaca derecha se va adquiriendo una posición antálgica 


\section{Consideraciones para la intervención quirúrgica frente a afecciones que provocan apendicitis aguda}

Vol. 3, núm. 3., (2019)

Ángela Lilibeth Peñafiel Bernal; Javier Alejandro Mera Cedeño; Andrea Isabel Vallejo

Maquilón; María Belén Meza Rivas

de semiflexión del muslo sobre el abdomen, la cual se hace más ostensible a medida que el proceso avanza.

- Pulso: Muy poco alterado al comienzo, va aumentando a medida que la temperatura asciende. Ciertas formas gangrenosas suelen acompañarse de bradicardia.

- Temperatura: En general no es elevada, sin embargo, en procesos flemonosos agudos o complicados se encuentra temperatura elevada. La diferencia apreciable entre la temperatura axilar y rectal se conoce con el nombre de disociación de temperaturas, se le concede cierto valor cuando es mayor de un grado. Su no presencia no invalida el diagnóstico.

Examen clínico.

- El paciente con apendicitis está agudamente enfermo, camina lentamente, muchas veces inclinado hacia delante protegiendo el lado derecho.

- Presenta facies de incomodidad o aprensión, tiende a retroceder cuando va a ser tocado. La cadera presenta ligera flexión.

- Presenta signos de deshidratación, dependiendo las horas de evolución del cuadro, fiebre y taquicardia (disociación esfigmotermica), palidez por dentro del surco nasogeniano.

- Se evalúa: ubicación del dolor, distensión abdominal, hiperestesia en la FID, presencia de defensa y contractura. Los ruidos hidroaéreos se encuentran disminuidos.

- Se utiliza la percusión suave para comprobar dolor a la descompresión (reacción peritoneal). La rigidez del psoas-iliaco denota inflamación del peritoneo posterior, por lo general a partir de la apendicitis retrocecal o formación de un absceso. Se buscan los signos del psoas y de Rovsing.

- Punto de McBurney. - Se obtiene presionando la fosa ilíaca derecha en un punto que corresponde a la unión del 1/3 externo con los 2/3 internos de una línea trazada de la espina ilíaca anterosuperior derecha hasta el ombligo. El dolor producido con esta maniobra es el encontrado con mayor regularidad. 


\section{Consideraciones para la intervención quirúrgica frente a afecciones que provocan apendicitis aguda}

Vol. 3, núm. 3., (2019)

Ángela Lilibeth Peñafiel Bernal; Javier Alejandro Mera Cedeño; Andrea Isabel Vallejo

Maquilón; María Belén Meza Rivas

- Signo de Blumberg. - Se obtiene presionando la pared de la fosa ilíaca derecha con toda la mano y retirándola bruscamente, el dolor que se produce es la manifestación de la inflamación del peritoneo apendicular y vecino.

En definitiva, el diagnóstico de apendicitis aguda puede ser hecho generalmente por el cuadro clínico. Cuando el diagnóstico es dudoso, una radiografía de abdomen simple de pie podría darnos algunos datos, como un nivel hidro aéreo en el cuadrante inferior derecho (asa centinela) o un fecalito calcificado que sería una evidencia de apendicitis, pero esto último sólo es visto en un 20$30 \%$ de casos. Otros signos son: escoliosis derecha, presencia de líquido peritoneal, masa de tejido blando y edema de pared abdominal, signos que son muy pobres como diagnóstico.

Una radiografía de tórax es indicada cuando se sospecha peritonitis primaria por neumonía. La ecografía se usa en casos dudosos, si el apéndice es identificado por ecografía se le considera inflamado, si no se le puede identificar se excluye la apendicitis. Es segura en excluir enfermedades que no requieren cirugía (adenitis mesentérica, ileítis regional, urolitiasis) así como diagnosticar otras entidades que sí requieren tratamiento quirúrgico, como embarazo ectópico, quistes de ovario, etc. Por su parte, la laparoscopía es el único método que puede visualizar el apéndice directamente, pero tiene la desventaja de invasividad. Esto requiere anestesia y de hecho es una operación que como cualquier otra tiene sus riesgos y complicaciones.

\section{Escalas Diagnosticas.}

Existen un gran número de instrumentos o escalas estructuradas que tratan de ayudar a detectar "posibles casos" de enfermedad. Las escalas por sí mismas no generan diagnósticos, sino que permiten seleccionar personas con puntuación alta en las que se sospecha la presencia de patología, lo que justifica la realización posterior de un estudio más profundo un especial cuidado. Además, estos instrumentos de medida sirven para completar una adecuada valoración, ya que refuerzan el juicio diagnóstico elaborado tras la entrevista clínica y la exploración física.

Todos estos instrumentos presentan limitaciones (sensibilidad y especificidad inferior al 100\%), por lo que no es factible ni recomendable utilizar escalas como uno método diagnóstico. En ningún caso sustituyen a la entrevista clínica o la exploración física, aunque son útiles como guía de la 


\section{Consideraciones para la intervención quirúrgica frente a afecciones que provocan apendicitis aguda}

Vol. 3, núm. 3., (2019)

Ángela Lilibeth Peñafiel Bernal; Javier Alejandro Mera Cedeño; Andrea Isabel Vallejo

Maquilón; María Belén Meza Rivas

misma y para apoyar el juicio clínico, así como también en el ámbito de la investigación clínica, además de servir para comprobar el efecto de distintas intervenciones en la evolución de la enfermedad.

El diagnóstico de apendicitis es difícil y existen algunas escalas que pretenden mejorar la exactitud diagnóstica. Ya que el diagnóstico temprano y correcto se considera la actuación clínica más significativa para reducir la morbimortalidad además de las complicaciones asociadas a esta enfermedad. No obstante, el proceso diagnostico constituye un desafío de significativa magnitud y exige la pericia de los médicos de los servicios de urgencias; razón ésta que motivó el surgimiento de distintas escalas; todas ellas, ideadas con el fin de aproximarse al diagnóstico; destacándose entre las mismas las siguientes:

\section{Escala RIPASA}

La cual fue realizado Brunei Durussalam en el Hospital Raja Isteri Pengiran Anak Saleha (RIPASA) en el 2010, Como una necesidad de adaptar las diferencias étnicas, alimentación, estilo de vida y al tipo de población (migración); reportándola con mejor sensibilidad (98\%) y especificidad (83\%). Sin embargo, en México solo existe un reporte de pacientes utilizando la escala RIPASA comparándola con la escala Alvarado la cual se realizó en el hospital general de México incluyendo 70 pacientes

De acuerdo al puntaje se sugiere el manejo:

- < 5 puntos (Improbable): observación del paciente y aplicar escala nuevamente en 1-2 h, si disminuye el puntaje, se descarta la patología, si éste aumenta se revalora con el puntaje obtenido.

- 5-7 puntos (Baja probabilidad): observación en urgencias y repetir escala en 1- 2 h o bien realizar un ultrasonido abdominal. Permanecer en observación.

- 7.5-11.5 puntos (Alta probabilidad de apendicitis aguda): valoración por el cirujano y preparar al paciente para apendicetomía, si éste decide continuar la observación, se repite en una hora. En caso de ser mujer valorar ultrasonido para descartar patología ginecológica. 


\section{Consideraciones para la intervención quirúrgica frente a afecciones que provocan apendicitis aguda}

Vol. 3, núm. 3., (2019) Ángela Lilibeth Peñafiel Bernal; Javier Alejandro Mera Cedeño; Andrea Isabel Vallejo Maquilón; María Belén Meza Rivas

- > 12 puntos (Diagnóstico de apendicitis): valoración por el cirujano para tratamiento o bien referirlo de ser necesario.

Escala SOLIS-MENA

En el año 2001 en el norte de México se realiza una escala para obtener un sistema puntuable basado en elementos clínicos y de laboratorio que pueda ser utilizado para incrementar el porcentaje de acierto, en el diagnostico apendicitis aguda el cual fue realizado en hospital general de zona (segundo nivel de atención) de torreón Coahuila, en el departamento de urgencias. La escala incluye elementos clínicos, así como de laboratorio con un total de 10 puntos como máximo de acuerdo al puntaje obtenido es la probabilidad diagnostica de apendicitis

Tabla $\mathbf{N}^{\circ}$ 2. Sistema de aplicación de resultados Escala Solís-mena

\begin{tabular}{|c|c}
\hline Puntos & Probabilidad de apendicitis \\
\hline $7-10$ puntos & $51.21 \%$ \\
\hline $6-5$ puntos & $45.53 \%$ \\
\hline $0-4$ puntos & $7.68 \%$ \\
\hline
\end{tabular}

Fuente: (Lenín 2016).

Escala de ALVARADO.

Con respecto a los exámenes complementarios para el diagnóstico de apendicitis aguda, en la biometría hemática completa (BHC) se encuentran los glóbulos blancos elevados en un 70\% - 90\% de los pacientes, con desviación a la izquierda en más del 75\% de los mismos de manera temprana cuando los glóbulos blancos aún son normales. La neutro filia incrementa hasta un $80 \%$ después de las primeras 24 horas. La probabilidad del diagnóstico correcto de apendicitis se incrementa cuando se hace hincapié en las manifestaciones clínicas específicas, las cuales se resumen en una escala de puntuación denominada la Escala de Alvarado. 


\section{Consideraciones para la intervención quirúrgica frente a afecciones que provocan apendicitis aguda}

Vol. 3, núm. 3., (2019)

Ángela Lilibeth Peñafiel Bernal; Javier Alejandro Mera Cedeño; Andrea Isabel Vallejo

Maquilón; María Belén Meza Rivas

\section{Conclusiones.}

Cuando se examina a un paciente con probable abdomen agudo, siempre ha de pensarse en apendicitis aguda, ya que ésta constituye en varios servicios de urgencia, la patología más frecuente y la primera causa de operaciones de emergencia. En las etapas iniciales del proceso es posible llegar en gran porcentaje al diagnóstico, pero las condiciones cambian cuando mayor es el tiempo de evolución y, sobre todo con la presentación de formas evolutivas y complicaciones subsecuentes.

El dolor abdominal es una patología muy frecuente de etiología multifactorial y la gran simuladora de muchas enfermedades, siendo una de ellas la apendicitis. En urgencias es una patología que se presenta muy frecuentemente, siendo relevante el diagnóstico oportuno en el primer contacto, a pesar de los múltiples medios auxiliares de diagnóstico (ultrasonido, tomografía, resonancia, etc.), hoy en día el diagnóstico de la apendicitis aguda es fundamentalmente clínico.

En razón de esto, se han ido desarrollando escalas diagnósticas, las cuales han sido ideadas con el fin de aumentar la certeza, su aplicación y mejora de la precisión diagnóstica, reduciendo con ello, las apendicetomías negativa o la presentación de complicaciones como lo mencionan Chong y Avi. Estas escalas y puntos de medición, ayudan a reducir gastos por hospitalización prolongada y estudios de imagen de mayor costo, así como incidir y disminuir riesgos de mortalidad y morbilidad.

Una de las escalas es la RIPASA desarrollada, en el sudeste de Asia en el hospital RIPAS tiene como propósito adecuar a pacientes con otro tipo de alimentación (alta en fibra) y que conlleva un factor protector, la cual ya fue validad y presenta una sensibilidad del $98 \%$ y especificidad del $83 \%$

a diferencia de la escala Solís - Mena realizada por médicos mexicanos con población del norte del país la cual fue realizada con 100 pacientes y de acuerdo al reporte de SOLIS Y COLS se presenta mayor sensibilidad y especificidad (sensibilidad 92\% especificidad 93\%) que la escala de ALVARADO, sin embargo esta escala no ha sido validada. 


\section{Consideraciones para la intervención quirúrgica frente a afecciones que provocan apendicitis aguda}

Vol. 3, núm. 3., (2019)

Ángela Lilibeth Peñafiel Bernal; Javier Alejandro Mera Cedeño; Andrea Isabel Vallejo

Maquilón; María Belén Meza Rivas

Por otra parte, se encuentran los sistemas clínicos de puntuación, los mismos son una herramienta económica y de rápida aplicación en los servicios de urgencias para descartar apendicitis aguda. Este sistema de puntuación es dinámico, lo que permite la observación y la reevaluación clínica de acuerdo a la evolución del paciente, tal y como lo establece la escala Solís mena, la cual presenta mayor exactitud diagnóstica que la escala RIPASA.

\section{Bibliografía.}

Arias, F. (2010). Paradigmas de la Investigación Científica. España: Editorial: Luces.

Castro, E. (2016). La Apendice y su comportamiento Agudo. Revista Médica de Buenos Aires: Estado actual de la apendicitis medigrafic., 17.

Cerpa, A. (2015). Factores de riesgo que inciden en las complicaciones de Apendicitis Aguda. Revista Médica de la Facultad de Medicina de la Universidad de Los Andes ULA, 14.

Dávila, N. (2012). Paradigmas de la Investigación Científica. Pereire, Colombia: Editorial: Las Brisas.

Lenín, J. (2017). Causas de la Apendicitis Aguda. Revista médica de costa rica y centroamerica, 28.

Penza, S. (2016). Diagnóstico de la Apendicitis Aguda. Revisión de Cirugía de la Universidad Centrooccidental Lisandro Alvarado UCLA, Lara, Venezuela., 22.

Selis, M. (2016). Complicaciones de la Apendicitis Aguda. Revista: Journal of Medicine Intensive., 11.

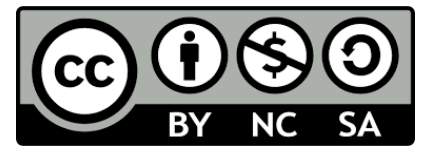

RECONOCIMIENTO-NOCOMERCIAL-COMPARTIRIGUAL

CC BY-NC-SA

ESTA LICENCIA PERMITE A OTROS ENTREMEZCLAR, AJUSTAR Y CONSTRUIR A PARTIR DE SU OBRA CON FINES NO COMERCIALES, SIEMPRE Y CUANDO LE RECONOZCAN LA AUTORÍA Y SUS NUEVAS CREACIONES ESTÉN BAJO UNA LICENCIA CON LOS MISMOS TÉRMINOS. 